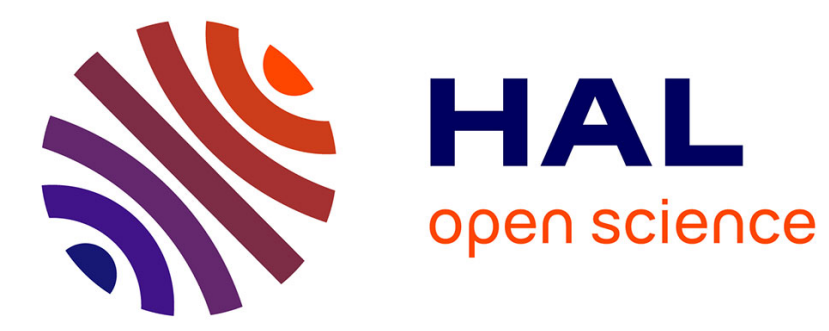

\title{
Nicolas Maisetti, Opération culturelle et pouvoirs urbains.
}

Jean-Christophe Sevin

\section{To cite this version:}

Jean-Christophe Sevin. Nicolas Maisetti, Opération culturelle et pouvoirs urbains.. 2015. halshs01954496

\section{HAL Id: halshs-01954496 \\ https://shs.hal.science/halshs-01954496}

Submitted on 9 Jan 2019

HAL is a multi-disciplinary open access archive for the deposit and dissemination of scientific research documents, whether they are published or not. The documents may come from teaching and research institutions in France or abroad, or from public or private research centers.
L'archive ouverte pluridisciplinaire HAL, est destinée au dépôt et à la diffusion de documents scientifiques de niveau recherche, publiés ou non, émanant des établissements d'enseignement et de recherche français ou étrangers, des laboratoires publics ou privés. 


\title{
Nicolas MAISETtI, Operation CULTURELLE ET POUVOIRS URBAINS
}

\author{
JeAN-ChRISTOPHE SEVIN
}

\begin{abstract}
Nicolas Maisetti, Opération culturelle et pouvoirs urbains. Instrumentalisation économique de la culture et luttes autour de Marseille-Provence Capitale européenne de la culture 2013, Paris, L'Harmattan, coll. « Questions contemporaines », 2014, 172 p., ISBN : 978-2-343-04931-1.
\end{abstract}

Dans un contexte postindustriel de reconversion économique des villes, les politiques culturelles longtemps séparées du domaine économique se sont transformées lorsqu'elles ont commencé à être envisagées comme un outil de régénération économique et urbaine ${ }^{1}$. L'évolution des Capitales Européennes de la Culture est à ce titre emblématique ${ }^{2}$. L'ouvrage de Nicolas Maisetti consacré au cas de Marseille-Provence Capitale Européenne de la Culture 2013, se concentre sur la trajectoire de la candidature et de la phase de préparation de l'opération. L'objectif étant d'analyser la transformation des pouvoirs urbains à travers l'étude de la dynamique des rapports entre acteurs politiques, économiques et culturels.

Dans une première partie, Nicolas Maisetti envisage les enjeux multi-scalaires de Marseille-Provence 2013, dans une conjonction de facteurs nationaux, régionaux et internationaux dont le dossier de candidature a su habilement tirer parti. Non seulement en mettant en avant le facteur économique consistant à faire de M-P 2013 un moyen de renforcer l'attractivité du territoire et de résorber les fractures territoriales au niveau métropolitain mais aussi en jouant sur une conjoncture géostratégique. En effet, la désignation de Marseille en septembre 2008 intervient quelques semaines après le lancement de l'Union pour la Méditerranée, le projet porté par le président Nicolas Sarkozy pour relancer le partenariat entre les pays riverains de la méditerranée. La candidature a ainsi plaidé un besoin de l'Europe pour Marseille " pour enrayer son déclin et rebondir économiquement " et un besoin de Marseille pour l'Europe "si elle veut mettre en œuvre une politique européenne efficace »(p. 20). L'auteur montre que l'habileté a consisté à " s'engouffrer dans la critique regrettant la relégation de la culture dans la coopération méditerranéenne » (p. 22) et à adopter en conséquence une rhétorique euro-méditerranéenne. II pointe le fait que cette propriété méditerranéenne de Marseille, convertie en ressource et mise en avant dans le dossier de candidature, est une construction rhétorique mouvante, une "stratégie argumentative » (p. 29) impliquant la mise en retrait de la dimension provençale et de son folklore. De même, la dimension cosmopolite valorisée dans le dossier, renvoie à une « interprétation enchantée de l'histoire des

\footnotetext{
${ }^{1}$ Cf. Ambrosino Charles et Guillon Vincent, «Gouverner, consommer et produire. Les trois mondes de la ville créative ", in Guy Saez et Jean-Pierre Saez (dir.), Les nouveaux enjeux des politiques culturelles. Dynamiques européennes, Paris, La Découverte, 2012, p. 95-105.

${ }^{2}$ García Beatriz, «Cultural Policy and Urban Regeneration in Western European Cities: Lessons from Experience, Prospects for the Future », Local Economy, 19/4, 2004, p. 312-326.
} 
migrations » (p. 30) qui jette un voile sur la relégation sociale et spatiale d'une partie importante de la population. C'est toute l'équivoque qui entoure la construction du récit euro-méditerranéen de Marseille-Provence 2013 : "contribue-t-il à accroitre le sentiment d'appartenance à un espace commun ? Parvient-il à traduire et à manifester une identité partagée ? Ou ne fait-il que la supposer en se saisissant de la Méditerranée comme un instrument qui apparait soudainement rentable pour remporter une compétition et accroître son attractivité ? » (p. 32) On pourrait évoquer une autre source d'équivoque que l'auteur ne relève pas; si d'un côté "Marseille n'est pas la Provence " dans le positionnement culturel de la candidature, d'un autre côté le positionnement économique plaide une meilleure intégration territoriale de Marseille dans la Provence et son aire métropolitaine. On peut aussi regretter que l'auteur ne s'intéresse pas à l'impact des révolutions arabes de 2011 sur le positionnement euro-méditerranéen de Marseille-Provence 2013.

La seconde partie se concentre sur la dynamique des rapports entre acteurs économiques, politiques et culturels dans l'économie générale de la candidature. Pour Maisetti, le rôle pilote des acteurs économiques dans la construction de la candidature marque leur retour dans les circuits de la production urbaine, face à la persistance d'une image dégradée associée au crime organisé et à des indicateurs économiques en décrochage par rapport à la moyenne nationale. Le déclencheur de cet " engagement territorial » (p. 63) est la publication par la DATAR en 2003 d'un classement des villes européennes dans lequel Marseille arrive en $23^{\mathrm{e}}$ position. Le nouveau président de la Chambre de Commerce prend alors appui sur ce classement pour «faire prendre conscience aux acteurs économiques et politiques du retard de la métropole marseillaise dans la concurrence européenne " (p. 69). Dans la perspective de faire entrer Marseille dans le Top 20 des métropoles européennes, le club ambition Top 20 créé par la Chambre de Commerce et d'Industrie Marseille-Provence et l'Union des Entreprise des Bouches du Rhône, saisit alors l'opportunité d'une candidature au titre de Capitale Européenne de la Culture comme outil de promotion du territoire avec "effet d'entrainement des grandes fonctions économiques créatrice de valeur » (p. 73).

Au-delà de l'union des acteurs politiques, économiques et culturels - le " jouer collectif » vanté par les premiers pour expliquer le succès de la candidature - une réalité plus contrastée nous est donnée à voir. Le rôle moteur des acteurs économiques dans la candidature a été accompagné par une mise à l'écart des acteurs politiques. Pour Maisetti, l'appropriation de l'opération par les acteurs politiques locaux était une condition du succès de la candidature qui avait besoin d'une légitimité démocratique, en même temps qu'elle était perçue comme source de perturbations et de remise en cause en raison des jeux d'acteurs politiques aux intérêts divergents. S'agissant plus particulièrement de Marseille, l'échec en 2003 de la candidature à la Coupe de l'America portée par la Mairie a conforté "la critique de l'amateurisme des fonctionnaires municipaux au regard des enjeux internationaux " (p. 79) et de la valorisation du territoire pour attirer des entreprises et des populations nouvelles ${ }^{3}$. Mais alors que les collectivités locales sont les plus gros financeurs de l'opération avec $85 \%$ du budget à leur charge, elles ont été en position d'accompagnement du projet. Quant aux acteurs culturels locaux, peu associés eux aussi à la candidature et à la gouvernance du projet, leurs positions sont diverses. Il y a celle de l'enrôlement stratégique, un positionnement qui peut être critique de l'instrumentalisation économique de leur activité tout en y trouvant dans le même temps la possibilité de la pérenniser. Des acteurs culturels exclus des jeux

\footnotetext{
${ }^{3}$ Cf. sur la Coupe de l'America : Samson Michel et Péraldi Michel, Gouverner Marseille. Enquête sur les mondes politiques marseillais, Paris, La Découverte, 2006.
} 
clientélistes, tout aussi critiques, peuvent néanmoins se réjouir des perturbations que M-P 2013 et sa direction externalisée a entraîné sur ces arrangements politiques. L'auteur repère un autre positionnement avec celui de la critique institutionnalisée, à l'image des acteurs du " off » qui ont été progressivement inclus dans le dispositif de Marseille-Provence 2013 et engagés dans une démarche de co-production de l'opération. Quant aux acteurs en position de critique radicale, ils dénoncent la disparition programmée de la ville prolétaire et populaire. Ici ce qui est en jeu est une lutte autour de la signification et du cadrage de l'opération: est-ce une opportunité à saisir, profitable pour la ville ; est-ce un écran de fumée masquant les vrais problèmes, un cheval de Troie des politiques d'ajustement structurel utilisant la culture comme véhicule ${ }^{4}$. Une analyse de la réception publique très instable et des conflits de cadrage dans cette phase préparatoire de l'opération viendrait utilement compléter ce travail.

La troisième partie de l'ouvrage se penche sur la gouvernance du projet. En 2008, suite à la désignation de Marseille-Provence 2013, une direction professionnalisée et externalisée ne dépendant pas des autorités politiques a été mise en place, ainsi que le recommande l'Union Européenne. Ceci a permis d'imposer un consensus s'appuyant sur les experts pour enclencher une coopération territoriale inédite entre les différentes collectivités locales partie prenante de Marseille-Provence 2013. Malgré des divergences autour du financement sur la question de l'équilibre entre "investissements consentis et retombées attendues»(p.146), ce "consensus opératoire " s'est ainsi manifesté dans une " coalition de causes " entre des acteurs ne partageant ni les mêmes intérêts ni les mêmes représentations qui sous-tendent le projet. Mais finalement l'auteur note que si la métropole fonctionnelle promue dans le dossier a ainsi servi à désamorcer les conflits politiques potentiels, la fragmentation politique et territoriale n'a pas été résolue pour autant.

On pourra regretter l'absence d'une mise en perspective avec d'autres tentatives antérieures visant à affronter la crise structurelle qui touche Marseille depuis la décolonisation, afin d'éclairer M-P 2013 avec une focale historique plus large et sans la grille de lecture de l'économie culturelle. De même, concernant l'intégration territoriale, l'impact de M-P 2013 gagnerait à être analysé en lien avec $\mathrm{d}^{\prime}$ autres tentatives dans le domaine des déplacements ou de l'enseignement supérieur ${ }^{5}$. Mais au final cet ouvrage, étayé par de nombreux entretiens avec des acteurs de l'opération au sein de diverses institutions, rend bien compte de la trajectoire du projet, de ses accrocs et soubresauts comme de la mobilité des positions en jeu dans la succession des différentes phases. II fournit un éclairage précieux sur la dynamique de constitution de Marseille-Provence 2013.

\section{REFERENCE ELECTRONIQUE}

Jean-Christophe Sevin, "Nicolas Maisetti, Opération culturelle et pouvoirs urbains ", Lectures [En ligne], Les comptes rendus, 2015, mis en ligne le 30 mars 2015 . URL : http://journals.openedition.org/lectures/17524

\footnotetext{
${ }^{4}$ Cf. Evans Graeme, "Hard-Branding the Cultural City - From Prado to Prada ", International Journal of Urban and Regional Research, 27/2, 2003, p. 417-440. Le récent documentaire de Nicolas Burlaud, La fête est finie, s'inspire du même mythe pour un propos allant dans ce sens. Cf. https://vimeo.com/113685047.

${ }^{5}$ Cf. Douay Nicolas, "L'émergence des politiques métropolitaines marseillaises : entre conflits et apprentissages », Cybergeo : European Journal of Geography [En ligne] http://cybergeo.revues.org/22347 ; DOI : 10.4000/cybergeo.22347.
} 